\title{
If I Touch I Like It! The Interplay Between Tactile Inputs and Gustatory Perceptions: An Abstract
}

\author{
Felipe Pantoja, Adilson Borges, Patricia Rossi, and Amanda Pruski Yamim
}

\begin{abstract}
Three studies test whether touching food items modulate consumers' purchase intentions and perceived taste during food sampling. In study 1, participants unobtrusively encouraged to touch (vs. not touch) a healthy food item (i.e. baby carrots) before tasting it declared higher purchase intentions and enhanced perceived taste when evaluating the product. In study 2, we replicated the results of study 1 with an unhealthy food item (i.e. nougat), so that touching (vs. not touching) the product resulted in better consumer responses towards the food (i.e. perceived taste and purchase intention). Finally, study 3 shows a boundary condition for the proposed effect. Specifically, the positive effect of touching is mitigated in sensoryoverloaded environments, suggesting that intense sensory conflicts might suppress touch prominence in an evaluative task. Therefore, the authors demonstrate that consumers' touch (vs. non-touch) in a food item enhances both gustatory perceptions and purchase intentions but only when other customer senses are not concurrently activated.
\end{abstract}

References Available Upon Request

\footnotetext{
F. Pantoja $(\varangle)$

IÉSEG School of Management, Paris, France

e-mail: f.pantoja@ieseg.fr
}

\author{
A. Borges • A.P. Yamim \\ NEOMA Business School, Reims, France \\ e-mail: adilson.borges@neoma-bs.fr; amanda.yamim@gmail.com \\ P. Rossi \\ IÉSEG School of Management (LEM-CNRS), Lille, France \\ e-mail: p.rossi@ieseg.fr
}

